\title{
Molecular Basis for the Interaction of Catalase with D-Penicillamine : Rationalization of some of its Deleterious Effects
}

\author{
Dominique Padovani* and Erwan Galardon
}

UMR 8601, LCBPT, CNRS-Université de Paris, 45 rue des Sts Pères, 75006 Paris, France

*Corresponding author. Email: dominique.padovani@parisdescartes.fr

\section{Graphical abstract}

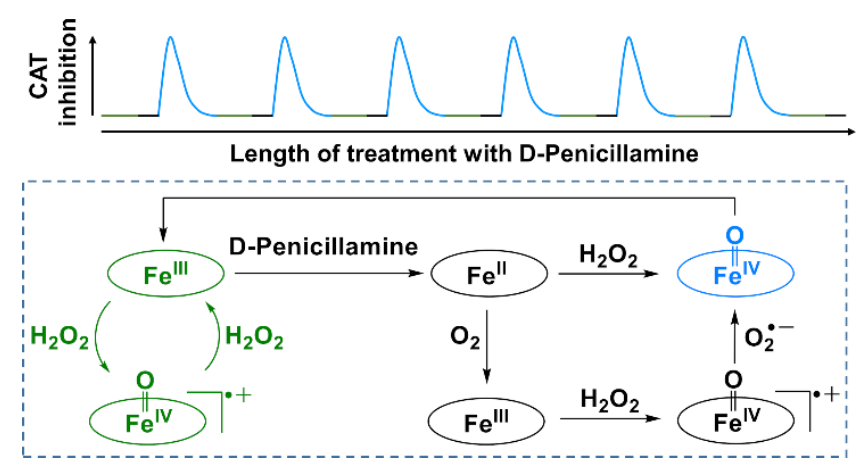




\section{Abstract}

D-penicillamine (D-Pen) is a sulfur compound used in the management of rheumatoid arthritis, Wilson's disease (WD), and alcohol dependence. Many side effects are associated with its use, particularly after long-term treatment. However, the molecular bases for such side effects are poorly understood. Based on the well-known oxidase activity of hemoproteins, and the participation of catalase in cellular $\mathrm{H}_{2} \mathrm{O}_{2}$ redox signaling, we posit that D-Pen could inactivate catalase, thus disturbing $\mathrm{H}_{2} \mathrm{O}_{2}$ levels. Herein, we report on the molecular bases that could partly explain the side effects associated with this drug compound, and we demonstrate that it induces the formation of compound II, a temporarily inactive state of the enzyme, through two distinct mechanisms. Initially, D-Pen reacts with native catalase and/or iron metal ions, used to mimic non heme iron overload observed in long-term treated WD patients, to generate thiyl radicals. These partake into a futile redox cycling, thus producing superoxide radical anions $\mathrm{O}_{2}{ }^{\bullet-}$ and hydrogen peroxide $\mathrm{H}_{2} \mathrm{O}_{2}$. Then, either $\mathrm{H}_{2} \mathrm{O}_{2}$ unexpectedly reacts with native CAT-Fe(II) to produce compound II, or both aforementioned reactive oxygen species intervene into compound II generation through compound I formation then reduction. These findings support evidence that D-Pen could perturb $\mathrm{H}_{2} \mathrm{O}_{2}$ redox homeostasis through transient but recurring catalase inactivation, which may in part rationalize some deleterious effects observed with this therapeutic agent, as discussed. 


\section{Introduction}

D-penicillamine (D-Pen) is an unnatural thiol mostly implicated in three types of reactions when used in a biological context: thiol-disulfide exchange, thiazolidine formation in the presence of carbonyl compounds, and metal chelation $(\mathbf{1 , 2})$. As a result, D-Pen is used as a therapeutic copper chelating agent in the treatment of Wilson's disease (WD), an autosomal recessive inherited copper metabolism disorder that results in severe defects such as oxidative tissue damage, liver damage (chronic hepatitis and cirrhosis), and ultimately death if untreated (3).

D-Pen is also used as an immunosuppressor in the treatment of rheumatoid arthritis (RA), a chronic inflammatory disease of the joints in which reactive oxygen species (ROS) as well as various enzymes released at the inflamed joints (e.g. heme-dependent myeloperoxidase, MPO) may contribute to the etiology of the disease (4-5). D-pen may exert a modulatory function on the oxidant-producing capacity of MPO by scavenging MPO-generated hypochlorous acid and by reacting with redox intermediates, thus contributing to superoxide radical anions $\left(\mathrm{O}_{2}{ }^{-}\right)$production and then inactive compound III (MPO-Fe(III)- $\left.\mathrm{O}_{2}{ }^{-*}\right)$ generation (6).

Last, D-Pen has been reported to act as a sequestration agent of acetaldehyde (ACD), a metabolite of ethanol generated either through the action of alcohol dehydrogenase in the liver or via the peroxidatic activity of catalase (CAT) in the brain (7-8). Brain derived ACD acts as a neuroactive agent in the central nervous system (9). As a result, strategies have been established for blocking ethanol metabolic pathways (e.g. inhibition of catalase bioactivity) and trapping ACD (e.g. sequestering of ACD by D-Pen through thiazolidine formation) (8-9).

Unfortunately, although the D-enantiomer does not display the acute toxicity encountered with the use of the L-enantiomer, a broad spectrum of toxicities has been reported for D-Pen particularly with increasing time of treatment (2-4). Thus, D-Pen exhibits severe secondary effects such as hepatotoxicity (10), immune disorders (11), and high incidence of neurological worsening (12-13). Additionally, hepatic non-heme iron overload occurs in patients presenting 
with WD during long-term treatment with D-Pen (14). This combination of copper and iron perturbed homeostasis may play a significant role in the pathogenesis of acute and chronic liver damage observed in animal models of WD (15). Also, a recent study suggests that D-Pen could selectively perturb hydrogen sulfide homeostasis, thus reducing vasodilation in mouse artoa rings, or exacerbating the inflammatory response in a mouse model of inflammation (16).

Noteworthy, some of D-Pen side effects could also be explicated by considering a D-Peninduced alteration of hydrogen peroxide $\left(\mathrm{H}_{2} \mathrm{O}_{2}\right)$ homeostasis. Indeed, while $\mathrm{H}_{2} \mathrm{O}_{2}$ is a wellrecognized signaling messenger (17), it is also well-known for its cytotoxic effects and its contribution to the etiology of immune diseases, its involvement in vasoconstriction, and its participation to a time-dependent increase in the cellular labile iron pool in neuronal cells (1820). On the basis of the well-known thiol oxidase activity of hemoproteins (21), including catalase (22), and the participation of catalase in cellular $\mathrm{H}_{2} \mathrm{O}_{2}$ redox signaling (23), we posit that D-Pen could induce catalase inactivation and thus disturb $\mathrm{H}_{2} \mathrm{O}_{2}$ homeostasis, which could play a key role in the reported cytotoxicity of D-Pen. Accordingly, we deciphered the reactivity of D-Pen with catalase.

We report here that catalase is indeed inactivated by D-Pen with a pharmacologically relevant relative $I_{50}$ value. The reactivity of catalase with $\mathrm{D}$-Pen first involves $\mathrm{D}$-Pen oxidation to its corresponding thiyl radical. The latter next enter a futile redox cycling that further facilitates the formation of compound II $(\mathrm{CAT}-\mathrm{Fe}(\mathrm{IV})=\mathrm{O})$, a temporarily inactive state of catalase. Interestingly, the presence of iron metal ions, used to mimic hepatic non-heme iron overload occurring in WD patients treated with D-Pen, has a significant effect on the inhibitory capacity of D-Pen, leading to a drastic drop in the relative $I_{50}$ value. Surprisingly, the generation of compound II occurs through two distinct mechanisms, one of which is unusual. Our findings support evidence that D-Pen could momentarily perturb $\mathrm{H}_{2} \mathrm{O}_{2}$ redox homeostasis through transient catalase inactivation, especially in contexts associated with disturbed metal homeostasis. These findings partly explain some deleterious side effects resulting from the use of D-Penicillamine as a therapeutic agent. 


\section{Experimental Procedures}

Materials - All chemicals were purchased from Sigma-Aldrich and used as-is. Bovine liver catalase and superoxide dismutase (SOD) from bovine erythrocytes were also purchased from Sigma-Aldrich. Hydrogen peroxide solution (9.79 M) TraceSELECT Ultra was purchased from Fluka. Nitrones used in this study were a kind gift from Dr. F. Peyrot (Université de Paris, France), with the exception of 5,5-dimethyl-1-pyrroline $\mathrm{N}$-oxide (DMPO) that was purchased from Sigma-Aldrich.

Enzymatic assays - CAT activity was measured on an Uvikon 941 spectrophotometer (Kontron) or a Cary 300 Scan (Varian) equipped with a temperature-controlled water bath $\left( \pm 0.1^{\circ} \mathrm{C}\right.$ ) by following the disappearance of $\mathrm{H}_{2} \mathrm{O}_{2}$ at $240 \mathrm{~nm}$ over $30-60 \mathrm{~s}$ at $25^{\circ} \mathrm{C}$, as previously described (22). Briefly, experiments were carried out in a $3 \mathrm{ml}$ quartz cuvette containing $11.7 \mathrm{mM} \mathrm{H}_{2} \mathrm{O}_{2}$ in $50 \mathrm{mM}$ phosphate buffer, $\mathrm{pH} 7.4 \pm 1 \mathrm{mM}$ diethylene triamine pentaacetic acid (DTPA). The reaction was initiated by adding 0.3-0.6 pmoles of CAT. The effect of D-Pen on the activity of CAT was determined from a solution of CAT $(30-60 \mathrm{nM})$ preincubated at $25^{\circ} \mathrm{C}$ for $120 \mathrm{~min}$ as a function D-Pen concentration $(0-5 \mathrm{mM}$ in the presence of DTPA or $0-0.5 \mathrm{mM}$ in the absence of DTPA) in $50 \mathrm{mM}$ phosphate buffer, $\mathrm{pH} 7.4 \pm 1 \mathrm{mM}$ DTPA. Noteworthy, CAT contains a $2.124 \pm 0.386$ molar excess of nonspecific iron metal ions, as previously determined (22). Then, $10 \mu \mathrm{l}$ of the reaction mixture was added to the $3 \mathrm{~mL}$ cuvette containing the $\mathrm{H}_{2} \mathrm{O}_{2}$ solution to initiate the reaction. Similar experiments were realized in the presence of $0.1 \mathrm{M}$ DMPO or $0.4 \mathrm{mM}$ bicyclo[6.1.0]nonyne $(\mathrm{BCN})$. The relative half inhibitory concentration $\left(\mathrm{IC}_{50}\right)$ values were obtained by plotting the relative activity of CAT as a function of D-Pen concentrations, and by fitting the data with a four-parameter logistic equation: $A=A_{\min }+\left(A_{\max }-A_{\min }\right) /\left(1+\left([D-P e n] / / C_{50}\right)^{n}\right)$, where $A_{\max }$ is the maximal activity of CAT (constrained at $100 \%), A_{\min }$ is the minimum activity achieved at saturating concentration of D-Pen and $n$ is the Hillslope that characterizes the slope of the curve at its midpoint. Each experiment was performed at least in triplicate. 
UV-visible Spectroscopy - UV-Visible spectra were recorded on an Uvikon 941 spectrophotometer (Kontron) equipped with a water bath $\left( \pm 0.1^{\circ} \mathrm{C}\right)$. UV-visible spectral changes over time induced by D-Pen reactivity with catalase were recorded as previously described (22) in a cuvette containing catalase $(3-5 \mu \mathrm{M})$ in $50 \mathrm{mM}$ phosphate buffer, $1 \mathrm{mM}$ DTPA pH 7.4 at $25^{\circ} \mathrm{C}$. The reaction was initiated by adding D-Pen (2 mM). The kinetic parameters associated with the reaction between catalase and D-Pen were obtained by plotting the relative absorbance at $404 \mathrm{~nm}$ (disappearance of native catalase) or $426 \mathrm{~nm}$ (appearance of compound II) as a function of time, and by fitting the data with a triple or double exponential function, respectively. Similar experiments were carried out in the presence of various nitrones such as 5-tert-butoxycarbonyl 5-methyl-1-pyrroline $\mathrm{N}$-oxide (BMPO, $10 \mathrm{mM}$ ), 5-diethoxyphosphoryl-5-methyl-1-pyrroline $\quad \mathrm{N}$-oxide $\quad$ (DEPMPO, $50 \quad \mathrm{mM}), \quad 5-$ diisopropoxyphosphoryl-5-methyl-1-pyrroline $\mathrm{N}$-oxide (DIPPMPO, $50 \mathrm{mM}$ ) or DMPO (100 $\mathrm{mM})$, increasing amount of SOD $(0-200 \mathrm{U})$ or $\mathrm{BCN}(0.4 \mathrm{mM})$. The kinetics for compound II reduction to native catalase were determined in separate experiments by adding buffer, DMPO (100 mM), BCN $(0.4 \mathrm{mM})$ or SOD $(200 \mathrm{U})$ to a reaction mixture containing catalase $(5 \mu \mathrm{M})$ that already reacted for 200-220 min with D-Pen (2 mM) in $50 \mathrm{mM}$ phosphate buffer, 1mM DTPA $\mathrm{pH} 7.4$ at $25^{\circ} \mathrm{C}$. The relative absorbance at $404 \mathrm{~nm}$ was then plotted as a function of time and the data were fit with a sigmoidal function: $A=A_{\min }+\left(A_{\max }-A_{\min }\right) /\left(1+e^{-(t-t 0.5) / n}\right)$, where $A_{\max }$ is the absorbance of native catalase (constrained at 1 ), $A_{\min }$ is the absorbance of catalase after 220 min reactivity with D-Pen, $t_{0.5}$ is the half-life of compound II, and $\mathrm{n}$ describes the steepness of the curve. The rate constant for compound II reduction $k_{\text {obs }}$ were then derived from $t_{0.5}$ using the linear relation: $t_{0.5}=\ln 2 / k_{\text {obs }}$.

Stopped-Flow UV-visible Spectroscopy - The inactivation kinetics with D-Pen were briefly analyzed on a sequential stopped-flow apparatus SFM-3 from Bio-Logic Science Instruments at ICMMO (Université Paris-Saclay, France). The following experiments were performed mainly to study the influence of SOD on compound I formation: catalase ( $4 \mu \mathrm{M}$ final) and DPen (2 mM final) solutions were mixed together in the absence or presence of $200 \mathrm{USOD}$ 
(final) in $50 \mathrm{mM}$ phosphate buffer, $1 \mathrm{mM}$ DTPA pH 7.4. The kinetic data were recorded over $300 \mathrm{~s}$ or $620 \mathrm{~s}$ and analyzed using the BIO-KINE software application.

HPLC-Mass spectrometry analysis - HPLC-MS (High Performance Liquid Chromatography coupled to Mass Spectrometry) spectra were recorded on a Thermo-Finnigan Surveyor equipped with a Satisfaction RP18AB $\mathrm{C}_{18} 3 \mu \mathrm{m}(15 \times 2 \mathrm{~mm})$ (Cluseau) and coupled to an ESI LCQ Advantage (Thermo Fisher Scientific). The running mixture was subjected to filtering on a Microcon filter unit of $10 \mathrm{kDa}\left(15 \mathrm{~min}, 4^{\circ} \mathrm{C}, 13,000 \mathrm{rpm}\right)$ and the analyses were conducted on the flow-through. The HPLC separation was achieved at $0.2 \mathrm{~mL} / \mathrm{min}$ using the following steps: 0-20 \% B (0-15 min), $20 \%$ B (15-25 min), 20-60 \% B (25-30 min), $60 \%$ B (30-50 min), $60-0 \%$ B (50-51 min), $0 \%$ B (51-60 min), with $A=0.1 \%$ formic acid in water (or $10 \mathrm{mM}$ ammonium acetate buffer, $\mathrm{pH} 4.6$ ) and $\mathrm{B}=$ acetonitrile. Under these conditions, the retention time for D-Pen disulfide and the adduct of D-Pen-BCN were $7.5 \mathrm{~min}$ and $27.7 \mathrm{~min}$, respectively.

EPR spectroscopy - EPR measurements were performed on a Bruker Elexsys 500 EPR spectrometer operating at X-band $(9.85 \mathrm{GHz})$ and equipped with a SHQ high-sensitivity cavity. Typical settings were: modulation frequency, $100 \mathrm{kHz}$; modulation amplitude $0.2 \mathrm{mT}$; receiver gain, $60 \mathrm{~dB}$; time constant, $40.96 \mathrm{~ms}$; conversion time, $41.04 \mathrm{~ms}$; sweep width, $15 \mathrm{mT}$ and sweep time, $42.02 \mathrm{~s}$. EPR spectra were recorded sequentially during the whole reaction course at $21^{\circ} \mathrm{C}$. Typical experiments were performed on a $50 \mu \mathrm{L}$ solution containing $50 \mu \mathrm{M}$ catalase, $2 \mathrm{mM}$ D-Pen and nitrones at $50 \mathrm{mM}$ (DEPMPO) or $200 \mathrm{mM}$ (DMPO) in $50 \mathrm{mM}$ phosphate buffer, $1 \mathrm{mM}$ DTPA $\mathrm{pH} 7.4$ at room temperature. Data acquisition and processing were performed using Bruker Xepr software. Appropriate control experiments (nitrone \pm catalase, D-Pen or potassium ferricyanide) were performed.

\section{Results}

Catalase is inhibited by D-penicillamine - At first, we monitored CAT activity as a function of the concentration of D-penicillamine (D-Pen) in the presence or absence of the chelating agent diethylene triamine pentaacetic acid (DTPA). In the presence of DTPA, CAT is partially 
inactivated by $D-P e n$ with a relative $\mathrm{IC}_{50}$ value of $191 \pm 26 \mu \mathrm{M}(\mathrm{n}=9 \pm \mathrm{SD})$ (Fig. 1A) as well as a bimolecular rate constant of $21.5 \pm 2.7 \mathrm{M}^{-1} \mathrm{~min}^{-1}$ at $25^{\circ} \mathrm{C}$ (Fig. S1A). The partial inactivation of CAT $(48.4 \pm 0.9 \%)$ supports half-site reactivity, as previously observed with aminotriazole (24) or some biological thiols (22). As observed, D-Pen is a better inhibitor than the latter that display relative $\mathrm{IC}_{50}$ values (obtained in similar conditions) of $0.59 \pm 0.07 \mathrm{mM}, 1.44 \pm 0.37 \mathrm{mM}$ and 21.2 $\pm 8.5 \mathrm{mM}$ for cysteine (Cys), homocysteine (HCys) or glutathione (GSH), respectively (22).
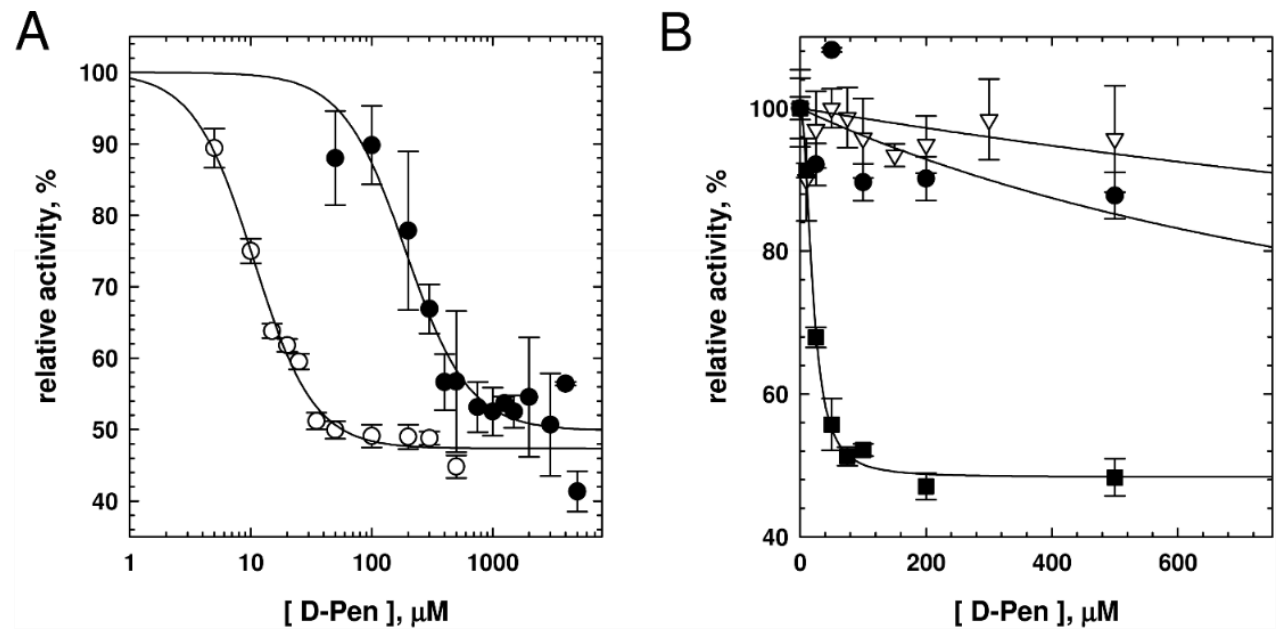

Figure 1 - Inhibition of catalase bioactivity by D-penicillamine. (A) Experiments showing the dependency of CAT activity on the concentration of D-Pen in the presence (black symbols) or absence (white symbols) of $1 \mathrm{mM}$ DTPA in $50 \mathrm{mM}$ phosphate buffer at $\mathrm{pH} 7.4$ and $25^{\circ} \mathrm{C}$. The plots were fitted with a four-parameter logistic equation and the relative $I C_{50}$ values $(n \geq 3 \pm S D)$ are reported in the text. (B) Dependency of CAT activity as a function of D-Pen concentration in the absence of DTPA (black squares) and in the presence of $0.1 \mathrm{M} \mathrm{DMPO}$ (black circles) or $0.4 \mathrm{mM} \mathrm{BCN}$ (white triangles) in $50 \mathrm{mM}$ phosphate buffer at $\mathrm{pH} 7.4$ and $25^{\circ} \mathrm{C}(\mathrm{n}=3 \pm \mathrm{SD})$.

In the absence of DTPA, the relative $\mathrm{IC}_{50}$ value drops to $10.6 \pm 0.5 \mu \mathrm{M}(\mathrm{n}=3 \pm \mathrm{SD})$ (Fig. 1A). This strongly suggests that sulfhydryl compounds and redox-active transition metal ions (in here iron metal ions) may cooperate to mediate the inactivation of catalase bioactivity. To determine which species is responsible for the aforementioned decrease in the relative $\mathrm{IC}_{50}$ value, we monitored the activity of CAT as a function of the concentration of D-Pen in the presence of iron metal ions (64-128 $\mathrm{nM})$, used to mimic hepatic non-heme iron overload, and various additives (Fig. 1B). D-Pen exhibits relative $\mathrm{IC}_{50}$ values greater than $1.25 \mathrm{mM}$ in the presence of the nitrone spin-trap 5,5-dimethyl-1-Pyrroline-N-Oxide (DMPO) or the bioconjugating agent 
bicyclo[6.1.0]nonyne $(\mathrm{BCN})$, suggesting that thiyl radicals and/or superoxide radical anions $\left(\mathrm{O}_{2}{ }^{--}\right)$promote the inhibition of CAT activity (Fig. 1B). In addition, superoxide dismutase (SOD) accelerates the initial inhibition velocity of CAT by D-Pen $\left(v_{i}=0.71 \pm 0.11 \mathrm{~min}^{-1}\right.$ or $v_{i}=1.10 \pm 0.15$ $\min ^{-1}(n=2 \pm S D)$ in the absence or presence of SOD, respectively) (Fig. S1B), strongly advocating for hydrogen peroxide $\left(\mathrm{H}_{2} \mathrm{O}_{2}\right)$ intervention during D-Pen-induced CAT inhibition. This observation is reminiscent of the inhibition of CAT bioactivity by biological thiols (22).

D-penicillamine induces compound II formation - Next, we monitored spectral changes over time when CAT is allowed to react with D-Pen in the presence of DTPA (Fig. 2A). The UV-
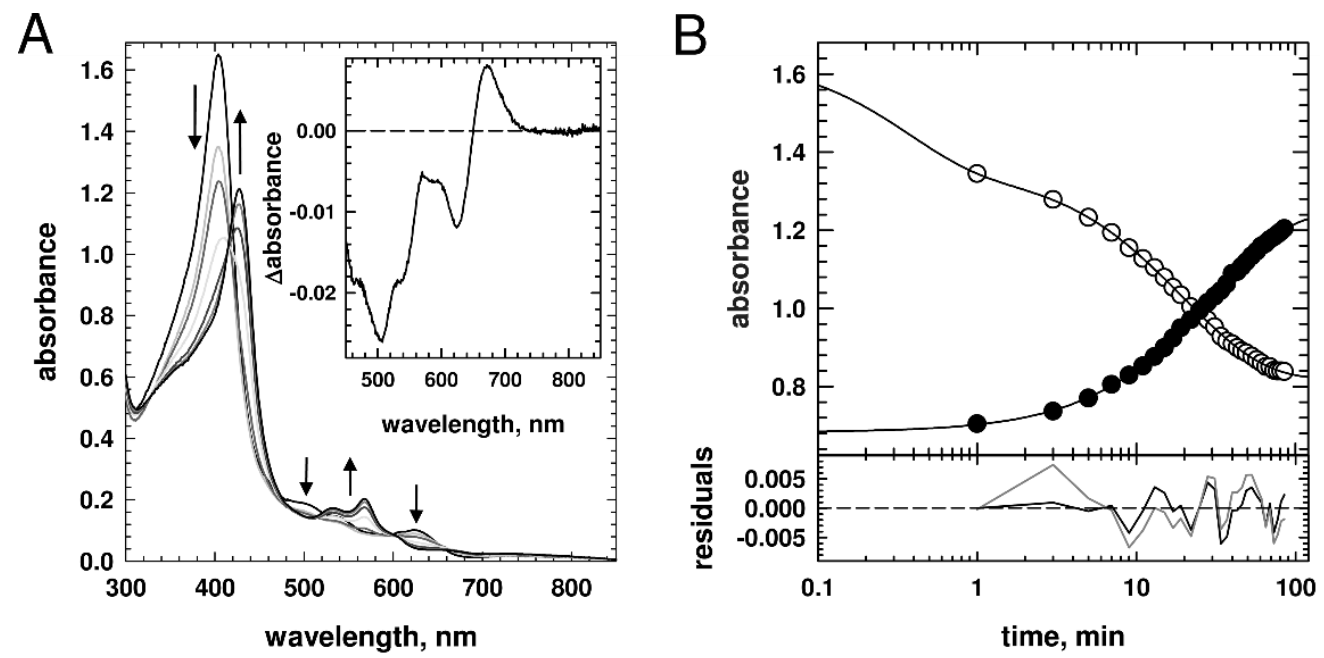

Figure 2 - D-penicillamine induces compound II formation. (A) Representative experiment showing the UV-visible spectral changes recorded over time $(0-89 \mathrm{~min})$ when catalase $(5 \mu \mathrm{M})$ reacts with D-Pen $(2 \mathrm{mM})$ in $50 \mathrm{mM}$ phosphate buffer, $\mathrm{pH} 7.4,1 \mathrm{mM}$ DTPA at $25^{\circ} \mathrm{C}$. Inset: Difference spectrum observed after catalase reacted for 1 min with D-Pen as in (A). The spectral peak around $660 \mathrm{~nm}$ is characteristic of compound I [CAT-Fe(IV)=O] $]^{+*}$. (B) Kinetics extracted from (A) at $404 \mathrm{~nm}$ (white symbols) and $426 \mathrm{~nm}$ (black symbols). The kinetic data at $404 \mathrm{~nm}$ were best fitted to a triple exponential function with $k_{\text {obs } 1}=2.83 \pm 0.34 \mathrm{~min}^{-1}, k_{\mathrm{obs} 2}=0.0996 \pm 0.0274 \mathrm{~min}^{-1}$ and kobs3 $=$ $0.0380 \pm 0.0073 \mathrm{~min}^{-1}$ as suggested by the residual plots below the graph. The dark and grey lines are the residual plots for a fit obtained with a triple or double exponential function, respectively. The kinetic data at $426 \mathrm{~nm}$ were best fitted to a double exponential function (dark trace) with $k_{0 b s} 1=$ $0.0613 \pm 0.0382 \mathrm{~min}^{-1}, k_{\mathrm{obs} 2}=0.0229 \pm 0.0105 \mathrm{~min}^{-1}$.

visible spectra recorded during the time course of the reaction clearly show the formation of several distinct species. The first transient species is formed within the minute, as observed by UV-visible spectroscopy (Fig. 2A) and confirmed by UV-visible stopped-flow spectroscopy (Fig. S2). This species exhibits distinct spectral features ( $\alpha$-band close to $660 \mathrm{~nm}$ ) characteristic of compound I, the porphyrin radical-ferryl state of catalase $[\mathrm{CAT}-\mathrm{Fe}(\mathrm{IV})=\mathrm{O}]^{++}$ (25). This intermediate is transformed into a second transient species typical of compound II, 
the one-electron oxidized ferryl state of catalase CAT-Fe(IV)=O (Soret band at $421, \alpha$-bands at 528 and $567 \mathrm{~nm}$ ) (22). Noteworthy, our rapid kinetic experiments failed to detect the appearance of a band at $411 \mathrm{~nm}$ or $414 \mathrm{~nm}$ that are characteristic of the CAT-Fe(II) and CAT$\mathrm{Fe}(\mathrm{II})-\mathrm{O}_{2}$ species, respectively (26). In addition, we did not observe any other intermediate species than those already described.

D-penicillamine oxidation mediates the formation of compound II through two distinct mechanisms - Next, we focused on obtaining insights into the formation of compound II by performing kinetic analyses. Kinetic analyses of CAT reactivity with D-Pen show that native CAT-Fe(III) disappearance recorded at $404 \mathrm{~nm}$ is best fitted with a triple exponential function, while CAT-Fe(IV)=O appearance recorded at $426 \mathrm{~nm}$ can be best fitted with a double exponential function (Fig. 2B). In addition, both rates and relative amplitudes at $426 \mathrm{~nm}$ are close to those observed for the last two stages at $404 \mathrm{~nm}$. These results thus strongly suggest that compound II, responsible for CAT inactivation, is generated through two apparent distinct routes respectively characterized by $k_{\mathrm{obs} 2}=0.0787 \pm 0.015 \mathrm{~min}^{-1}$ and $k_{\mathrm{obs} 3}=0.0443 \pm 0.0110 \mathrm{~min}^{-}$ $1(\mathrm{n}=7 \pm$ SD) (Table I).

Table I - Rate constants for the reactivity of catalase with D-penicillamine at $25^{\circ} \mathrm{C}$

\begin{tabular}{|c|c|c|c|c|}
\hline \multirow{2}{*}{ *Additives } & \multicolumn{2}{|c|}{$\mathrm{Fe}(\mathrm{IV})=0$ formation } & \multicolumn{2}{|c|}{$\mathrm{Fe}(\mathrm{IV})=0$ reduction } \\
\hline & $k_{\mathrm{obs} 2}, \min ^{-1}$ & $k_{\mathrm{obs} 3}, \min ^{-1}$ & $t_{1 / 2}, \min$ & $k_{\text {obs }}, \min ^{-1}$ \\
\hline None & $0.0787 \pm 0.015$ & $0.0443 \pm 0.0110$ & $496 \pm 14$ & $(1.39 \pm 0.05) \times 10^{-3}$ \\
\hline DMPO & †ND & ND & $2.1 \pm 0.4$ & $0.33 \pm 0.06$ \\
\hline $\mathrm{BCN}$ & ND & ND & $94 \pm 8$ & $(7.37 \pm 0.63) \times 10^{-3}$ \\
\hline SOD & $0.262 \pm 0.047$ & $0.0341 \pm 0.0031$ & $205 \pm 6$ & $(3.38 \pm 0.10) \times 10^{-3}$ \\
\hline
\end{tabular}

${ }^{*}$ Additives: $0.1 \mathrm{M} \mathrm{DMPO}, 0.4 \mathrm{mM}$ BCN or $200 \mathrm{U}$ SOD.

† ND: Not Detected. Compound II formation is impaired.

Noteworthy, the preferential route depicted by $k_{\text {obs } 3}\left(\Delta A_{3}=(1.54 \pm 0.42) \times \Delta A_{2}, n=7 \pm S D\right)$ exhibits a rate close to the ones registered for CAT- $\mathrm{Fe}(\mathrm{IV})=\mathrm{O}$ generation with similar concentrations of biological thiols, thus suggesting that this pathway may be associated with compound II production through compound I reduction by superoxide radical anions (22). To better decipher 
bioRxiv preprint doi: https://doi.org/10.1101/2021.09.16.460603; this version posted September 17, 2021. The copyright holder for this preprint (which was not certified by peer review) is the author/funder, who has granted bioRxiv a license to display the preprint in perpetuity. It is made available under aCC-BY-NC 4.0 International license.

the two distinct mechanisms responsible for compound II formation, we performed similar experiments to those described above in the presence of various additives, as detailed below.

Intervention of D-penicillamine-derived thiyl radicals during compound II formation - The reaction between native catalase and D-penicillamine begins with the reduction of CAT-Fe(III) by D-Pen (Fig. 3). This process should initially occur through the formation of the Low Spin

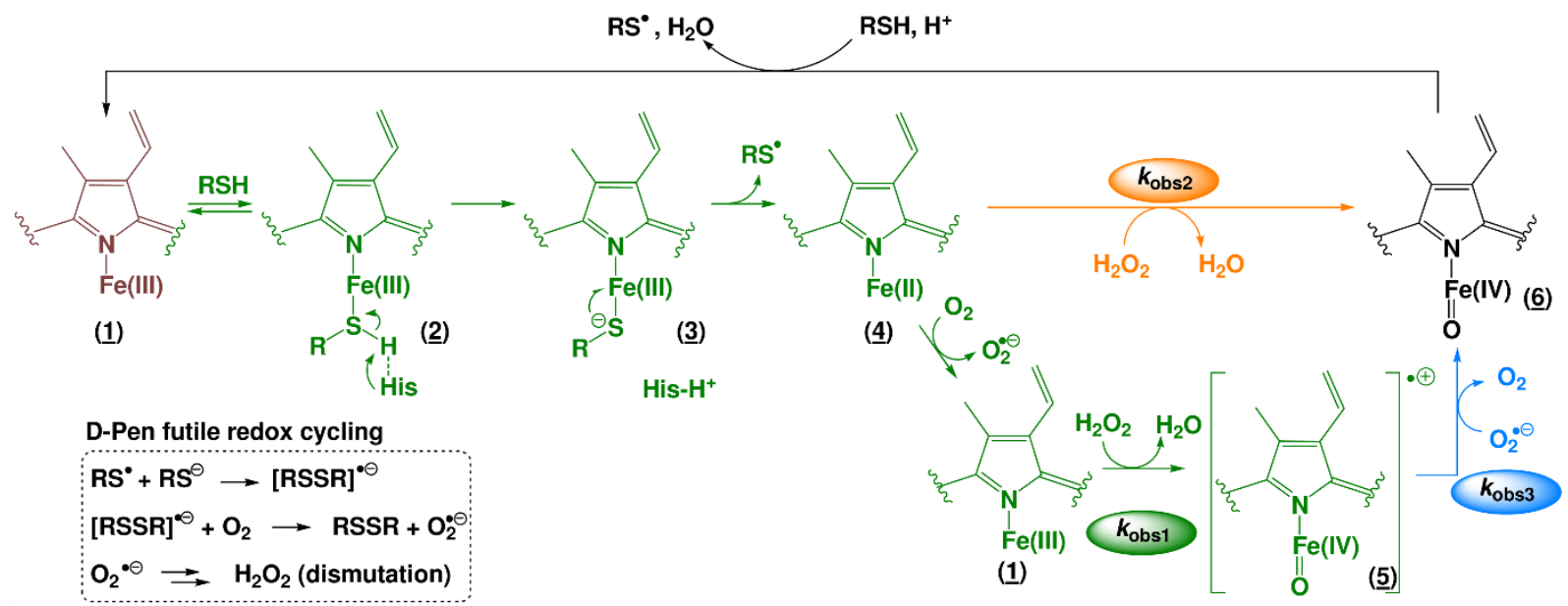

Figure 3 - Mechanisms for the reversible formation of compound II induced by D-penicillamine. The reaction between native CAT-Fe(III) and D-Pen (RSH) starts with the reduction of CAT-Fe(III) by D-Pen (steps (1)-(4)) through the generation of the Low Spin iron-complex CAT-Fe(III)-RSH (2), the deprotonation of the bound D-Pen by the distal histidine to generate an unstable CAT-Fe(III)-RScomplex ( $\underline{3})$ that is rapidly converted to reduced CAT-Fe(II) (4) along with a D-Pen-derived thiyl radical RS: The latter enters in a futile redox cycle described in the frame. At this point, CAT-Fe(II) (4) can be oxidized by molecular oxygen to CAT-Fe(III) (1) , which then reacts with $\mathrm{H}_{2} \mathrm{O}_{2}$ to form compound I (ㅁ). Noteworthy, the rate $k_{\text {obs } 1}$ measured at $404 \mathrm{~nm}$ during CAT reactivity with D-Pen (Fig. 2) corresponds to

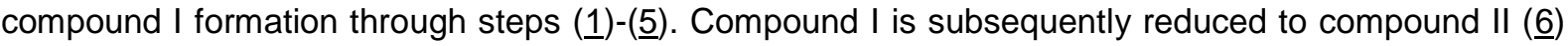
by superoxide radical anions with a rate $k_{o b s}$. Alternatively, compound II can be unexpectedly produced from the reaction between CAT-Fe(II) (4) and $\mathrm{H}_{2} \mathrm{O}_{2}$ at a rate $k_{0 b s}$ that exhibits sensitivity to superoxide dismutase (SOD). Last, compound II (ㅁ) slowly decays back to native CAT-Fe(III) (1) with a rate also sensitive to SOD.

iron-complex CAT-Fe(III)-D-Pen, then the deprotonation of the bound D-Pen by the distal histidine followed by the rapid reduction of CAT-Fe(III) to CAT-Fe(II) along with a D-Pen derived thiyl radical generation. As observed by UV-visible spectroscopy (Fig. 2A), both aforementioned CAT intermediates are not detected during the time course of CAT-Fe(IV)=O formation. So we focused on demonstrating the production of D-Pen derived radicals during the generation of compound II. Accordingly, we first examined spectral changes over time during the reactivity of CAT and D-Pen with $\mathrm{BCN}$, with subsequent analysis of the reaction 
mixture by HPLC-MS/MS, or with various nitrone spin trapping agents (DMPO, BMPO, DEPMPO or DIPPMPO) (Table I, Fig. 4A-C and S3A).
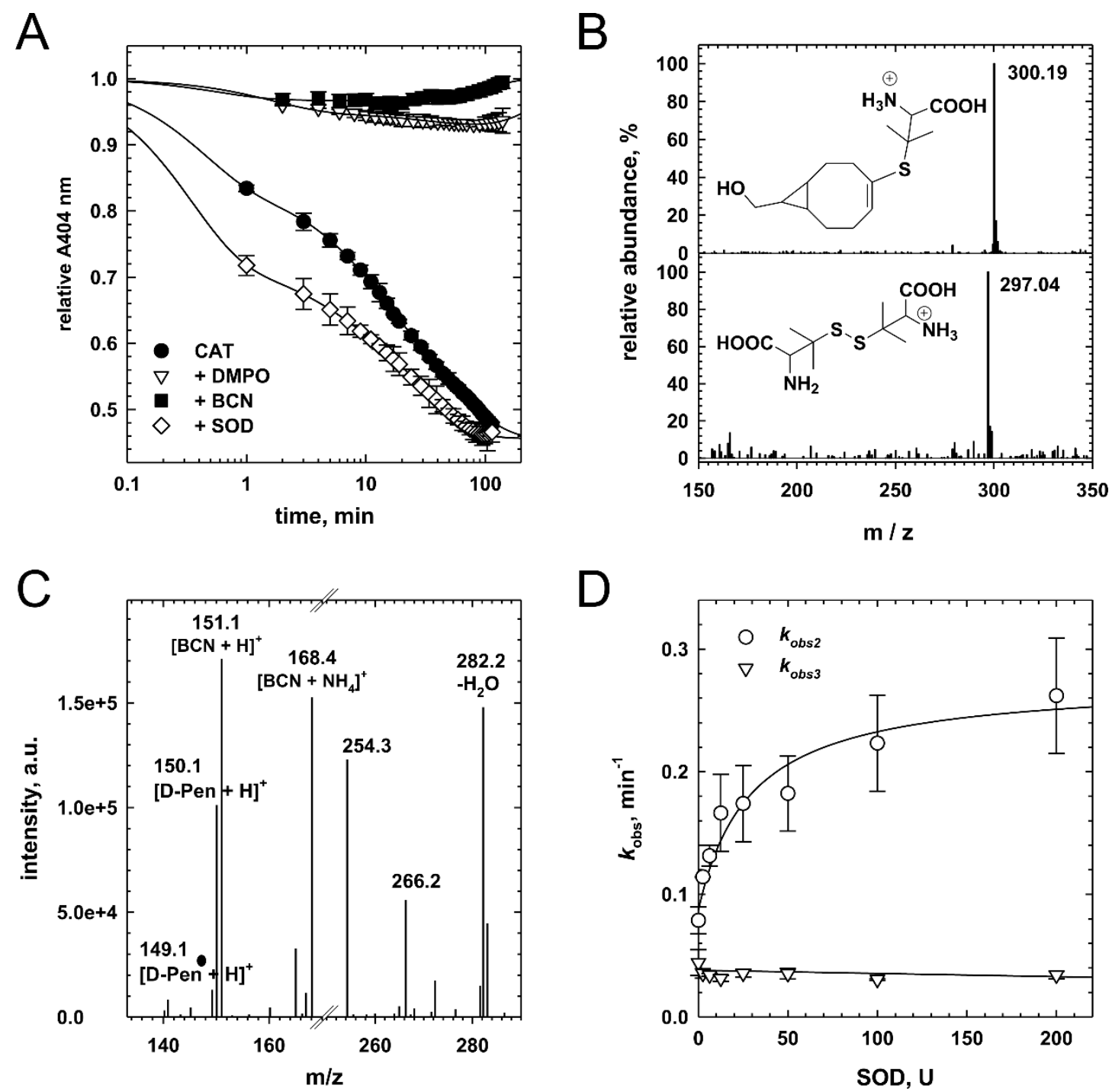

Figure 4 - Sulfur and oxygen reactive species intervene during compound II formation. (A) Representative effect of various additives (100 mM DMPO, $0.4 \mathrm{mM} \mathrm{BCN}$ or $200 \mathrm{U}$ SOD) on the kinetics of compound II formation during the reactivity of CAT $(5 \mu \mathrm{M})$ with D-Pen $(2 \mathrm{mM})$ in $50 \mathrm{mM}$ phosphate buffer, $1 \mathrm{mM}$ DTPA at $\mathrm{pH} 7.4$ and $25^{\circ} \mathrm{C}(\mathrm{n}=2 \pm \mathrm{SD})$. The kinetics for compound II formation in the absence of any additive (black circles) is shown for comparison. (B) Mass spectra (ESI+) of the BCN adduct (top) and the D-Pen disulfide (bottom) formed during the reactivity of catalase (4 $\mu \mathrm{M})$ with $\mathrm{D}$ Pen $(2 \mathrm{mM})$ in the presence of $0.4 \mathrm{mM} \mathrm{BCN}$ in $50 \mathrm{mM}$ phosphate buffer, $1 \mathrm{mM}$ DTPA at pH 7.4 and $25^{\circ} \mathrm{C}$. (C) MS-MS spectrum (ESI+) of the molecular ion with a $\mathrm{m} / \mathrm{z}=300.2$. The molecular ions at $\mathrm{m} / \mathrm{z}$ $=266.2$ and 254.3 result formally from the loss of $\mathrm{H}_{2} \mathrm{O}_{2}$ and a decarboxylation concomitant with the introduction of an unsaturation, respectively. The other main molecular ions are identified on the MSMS spectrum. (D) Effect of increasing amount of superoxide dismutase (0-200 U) on the rates of compound II formation during the reaction of CAT $(5 \mu \mathrm{M})$ with D-Pen $(2 \mathrm{mM})$ in $50 \mathrm{mM}$ phosphate buffer, $1 \mathrm{mM}$ DTPA at $\mathrm{pH} 7.4$ and $25^{\circ} \mathrm{C}$. The rates $k_{\mathrm{obs} 2}$ (white circles) and $k_{\mathrm{obs}}$ (white triangles) have been extracted from the absorbance at $404 \mathrm{~nm}$ as described in Figure 2 ( $n \geq 2 \pm S D$ ).

The comparative kinetic studies realized in the presence of BCN or DMPO (Table I, Fig. 4A)

or other nitrone spin trapping agents (Fig. S3A) clearly show that compound II formation is 
greatly impaired, thus strongly advocating for D-penicillaminyl radical intervention during CAT$\mathrm{Fe}(\mathrm{IV})=\mathrm{O}$ generation. The HPLC-MS/MS analysis of the reaction mixture obtained in the presence of $\mathrm{BCN}$ reveals the presence of a molecular ion at $\mathrm{m} / \mathrm{z}=300.19\left[\mathrm{M}+\mathrm{H}^{+}\right](\mathbf{F i g} . \mathbf{4 B})$ that corresponds well to a BCN-D-Pen adduct (Fig. 4C). This adduct, not detected in the absence of the enzyme, results from the addition of D-Pen-derived thiyl radicals to $B C N$ according to the thiol-yne reaction (27). Its presence in the reaction mixture thus clearly confirms the formation of D-penicillaminyl radicals through the oxidation of D-Pen by native CAT-Fe(III). Regrettably, even if the nitrone spin trapping agent DEPMPO strongly decelerates the rate of compound II formation (Fig. S3A) and the related reagent DMPO abolished CAT-Fe(IV)=O formation (Fig. 4A), we were unable to record any DMPO- or DEPMPO-D-Pen adducts by RT EPR spectroscopy, as observed with other biological thiols (22).

Intervention of reactive oxygen species during compound II generation - Once formed, the DPen-derived thiyl radicals should enter into a futile redox cycle and partake into the production of reactive oxygen species $\left(\mathrm{O}_{2}{ }^{-*}\right.$ and $\left.\mathrm{H}_{2} \mathrm{O}_{2}\right)$ along with D-Pen disulfide formation (Fig. 3). We thus analyzed by HPLC-MS the D-Pen derivatives formed during D-Pen reactivity with native CAT. Our analysis shows the presence of a molecular ion at $\mathrm{m} / \mathrm{z}=297.04\left[\mathrm{M}+\mathrm{H}^{+}\right]$that corresponds well to D-Pen disulfide (Fig. 4B). This observation confirms the intervention of Dpenicillaminyl radicals into a futile redox cycle that will in fine produce $\mathrm{O}_{2}{ }^{--}$then $\mathrm{H}_{2} \mathrm{O}_{2}$ (Fig. 3), as observed during D-Pen-dependent copper-catalyzed $\mathrm{H}_{2} \mathrm{O}_{2}$ generation (28-30).

Then, the formation of compound II can occur via two different likely scenarios (Fig. 3). In the first pathway, compound II may be generated from the reaction between CAT-Fe(II) and $\mathrm{H}_{2} \mathrm{O}_{2}$, a mechanism observed during the non-enzymatic heme degradation by reactive oxygen species (31) and the heterolytical cleavage of $\mathrm{H}_{2} \mathrm{O}_{2}$ by ferrous myeloperoxidase (32) or ferrous lactoperoxidase (33). As noted above, the second one may be associated with compound II production via a similar mechanism than that observed with biological thiols, i.e. compound I reduction by superoxide radical anion (22). To discriminate between both pathways and correctly attribute the observed kinetic parameters to one mechanism or the other, we 
performed comparative kinetic studies of compound II formation in the presence of SOD, which should favor the formation of compound II through the first pathway.

At first, we examined the reactivity of CAT and D-Pen over time in the presence of $200 \mathrm{U}$ SOD. The presence of SOD into the reaction mixture significantly accelerates compound I formation, as observed by UV-visible stopped flow spectroscopy (Fig. S2A), increases 3.3-fold the rate of compound II formation $k_{\mathrm{obs} 2}$ and slightly decreases the rate of compound II generation $k_{\text {obs } 3}$ (Table I, Fig. 4A). Next, we performed similar comparative kinetic studies in the presence of increasing amount of SOD. As reported in Fig. 4D, while $k_{\text {obs2 }}$ exhibits hyperbolic dependence on SOD amount $\left(k_{\min }=0.087 \pm 0.010 \mathrm{~min}^{-1}, k_{\max }=0.275 \pm 0.028 \mathrm{~min}^{-1}\right.$ and $[S O D]_{50}=28.9 \pm 10.1$ $U), k_{\text {obs3 }}$ displays a linear dependence on it (slope $\left.=(-2.6 \pm 1.8) \times 10^{-5}\right)$. Taken together, these results imply that $k_{\text {obs2 }}$ could be attributed to the formation of compound II through CAT-Fe(II) reactivity with $\mathrm{H}_{2} \mathrm{O}_{2}$, which is unusual and has never been demonstrated for CAT, and $k_{\text {obs } 3}$ could be associated with compound II formation through the reduction of compound I by $\mathrm{O}_{2}{ }^{\circ-}$, as observed with some biological thiols (22). Surprisingly, the amount of CAT-Fe(IV)=O generated through each respective pathway, represented here by the ratio $\Delta \mathrm{A} 2$ over $\Delta \mathrm{A} 3$, displays a bell-shaped curve as a function of SOD amount (Fig. S2B). Since the first pathway depends on $\mathrm{H}_{2} \mathrm{O}_{2}$ reactivity with CAT- $\mathrm{Fe}(\mathrm{II})$ and the second pathway relies on compound I reduction by $\mathrm{O}_{2}{ }^{\circ}$, we would have expected to observe a linear or hyperbolic dependence of the ratio $\Delta A_{2} / \Delta A_{3}$ as a function of SOD amount. Accordingly, this result implies that the proportion of compound II generated through each mechanism is finely adjusted by the amount of $\mathrm{O}_{2}{ }^{*-}$ vs $\mathrm{H}_{2} \mathrm{O}_{2}$ produced in the reaction mixture. It also suggests that the quantity and/or the flux of $\mathrm{H}_{2} \mathrm{O}_{2}$ modulates the selectivity of CAT-Fe(II) toward $\mathrm{H}_{2} \mathrm{O}_{2}$ or $\mathrm{O}_{2}$ oxidation.

Compound II is unstable in the absence of thiyl radicals and reactive oxygen species - Next, we recorded the reactivity of catalase with D-penicillamine over a long period of time to determine if CAT-Fe(IV)=O is reduced back to native CAT-Fe(III), as observed with Cys or GSH, or is converted to another species, as detected with HCys (22). As reported in Table I 
and Fig. S3B, compound II slowly decays back to CAT-Fe(III) with a rate of $(1.37 \pm 0.05) \times 10^{-}$ ${ }^{3} \mathrm{~min}^{-1}$. Noteworthy, the rate of decay to native catalase probably depends on a cycling between CAT-Fe(III) and compound II. If so, the more D-Pen-derived thiyl radicals and/or reactive oxygen species present in the reaction mixture, the longer will compound II be the dominating intermediate. To determine if that is the case we performed similar kinetic studies in the presence of various additives acting on the depletion of thiyl radicals or reactive oxygen species in the reaction mixture (Table I, Fig. S3B). The nitrone spin trap DMPO accelerates more than 230 -fold the reduction of compound II to native CAT-Fe(III) with a $k_{\text {obs }}$ of $(330 \pm 2)$ $\times 10^{-3} \mathrm{~min}^{-1}$, while $\mathrm{BCN}$ or SOD have a significant but less effect on its decay with $k_{\text {obs }}$ of $(7.42 \pm 0.72) \times 10^{-3} \mathrm{~min}^{-1}$ and $(3.32 \pm 0.11) \times 10^{-3} \mathrm{~min}^{-1}$, respectively. These results thus confirm our assumptions.

\section{Discussion}

We investigated here the reactivity of catalase toward D-penicillamine, a thiol that is potent in treating Wilson's disease, rheumatoid arthritis and alcohol dependence. The results of our studies offer a clear basis for understanding the mechanism whereby D-penicillamine reversibly inhibits catalase, hence possibly affecting various cellular processes through disturbance of $\mathrm{H}_{2} \mathrm{O}_{2}$ levels. Thus, our data indicate that complex electron transfer reactions take place between catalase and/or non-heme iron, D-penicillamine and oxygen, promoting the generation of $\mathrm{CAT}-\mathrm{Fe}(\mathrm{IV})=\mathrm{O}$, a temporarily inactive state of the enzyme, as well as reactive oxygen species and thiyl radical intermediates.

Numerous studies demonstrated that CAT bioactivity is either inhibited through the formation of 6-coordinate Low Spin (e.g. with cyanide) or 5-coordinate High Spin (e.g. with formate) heme-iron-complexes, the production of an unclear type I inactive catalase (e.g. with 2mercaptoethanol), the generation of sulfheme (e.g. with homocysteine) or compound II (e.g. with cysteine or glutathione) $(22,34-37)$. As reported in here, our data clearly indicate that CAT bioactivity is inhibited by D-Pen through the generation of compound II, as observed with Cys 
and GSH (22). However, D-Pen is a better inhibitor than biological thiols in term of half maximal inhibitory concentration $\mathrm{IC}_{50}$. This may be due to D-Pen higher relative hydrophobicity in comparison to the aforementioned biological thiols, as shown by their respective partition coefficient ClogP (Chemdraw 19.1, Perkin Elmer) of $-3.293,-3.678,-8.589$ or -2.585 for Cys, HCys, GSH and D-Pen, respectively. As a result, D-Pen could have better access to catalase active site. In addition, the lower $\mathrm{p} K_{\mathrm{a}}$ of the thiol group in D-Pen compared to the other aforementioned thiols could also account for these results. Accordingly, the relative reactivity of D-Pen and biologically important thiols with $\mathrm{O}_{2}{ }^{\bullet}$ or $\mathrm{H}_{2} \mathrm{O}_{2}$ is inversely related to their $\mathrm{p} K_{\mathrm{a}}(\mathbf{3 8})$. Noteworthy, the relative $\mathrm{IC}_{50}$ values exhibited by D-Pen, in particular the one displayed in the presence of iron metal ions used to mimic non heme iron overload, fall within the range of Dpenicillamine concentration observed in plasma of patients receiving the drug (30).

Usually, at low levels of $\mathrm{H}_{2} \mathrm{O}_{2}$ and in the presence of one-electron donors (for example biological thiols, phenols, aromatic amines, $\mathrm{NO}$ or $\mathrm{O}_{2}{ }^{\circ}$ ), compound I may go through a oneelectron reduction towards the temporarily inactive state of the enzyme compound II, which converts back to native catalase over a second one-electron reduction step $(22,36,39)$. Alternatively, compound II could be transformed into compound III, an oxyferrous state (CAT$\mathrm{Fe}(\mathrm{II})-\mathrm{O}_{2}$ ) of the enzyme that could go back to native CAT-Fe(III) along with $\mathrm{O}_{2}{ }^{\bullet-}$ release. As expected, the former scenario is also followed in the presence of D-Pen, without the detectable presence of compound III. However, based on experiments carried out in the presence of SOD that accelerates (hyperbolic dependence, Fig. 4D) and increases (bell-shape curve, Fig. S2B) the formation of compound II, we also demonstrated that a surprising involvement of CAT$\mathrm{Fe}$ (II) reactivity with $\mathrm{H}_{2} \mathrm{O}_{2}$ promotes compound II formation and competes with the classical pathway.

Previous investigations showed that the ferrous form of catalase is normally inaccessible by conventional reduction techniques of native catalase $(\mathbf{3 6})$ and requires the three-electron reduction of compound I, for example by azide that promotes azydil radical formation and NOferrocatalase generation $(\mathbf{4 0 , 4 1 )}$. Nevertheless, the present study underlines again the unique 
ability of thiols to reduce CAT-Fe(III) to CAT-Fe(II) (22). Also, contrary to some hemeperoxidase systems known to generate compounds II by the two-electron oxidation of their ferrous form by $\mathrm{H}_{2} \mathrm{O}_{2}(\mathbf{3 2}, \mathbf{3 3})$, the ferrous form CAT-Fe(II) obtained by reduction of native catalase with biological thiols (Cys, HCys or GSH) rapidly decays back to native catalase by reacting with molecular oxygen rather than yielding compound II (22). Therefore, D-Pen induces a new type of reactivity for ferrous catalase, which was initially proposed to explain the mechanism of decomposition of hydrogen peroxide by catalase (42) but was quickly dismissed (43). This observation may be explained by the effect of substituents on the stability of sulfur-centered radicals $(\mathbf{4 4 , 4 5})$ resulting from the reaction between the various thiols and native catalase. Accordingly, D-penicillamine-derived thiyl radicals could exhibit a gain in stability compared to thiyl radicals derived from biological thiols that could promote the participation of D-penicillaminyl radicals in the futile redox cycle described in Fig. 3 and therefore enhance the production of superoxide. Additionally, Winterbourn et al showed that D-Pen produces more hydrogen peroxide upon reaction with superoxide than other biologically important thiols (38). The creation of a greater flux and/or amount of $\mathrm{O}_{2}{ }^{-*}$ then $\mathrm{H}_{2} \mathrm{O}_{2}$ in the presence of D-penicillamine could thus explain its surprising capacity to participate in the formation of compound II by a new mechanistic avenue, the two-electron oxidation of ferrous catalase. Thus, it appears that the reactivity of catalase with biologically relevant thiols is strongly tuned by their structure, in which slight changes result in diverse reactivities, as illustrated in Fig. 5.

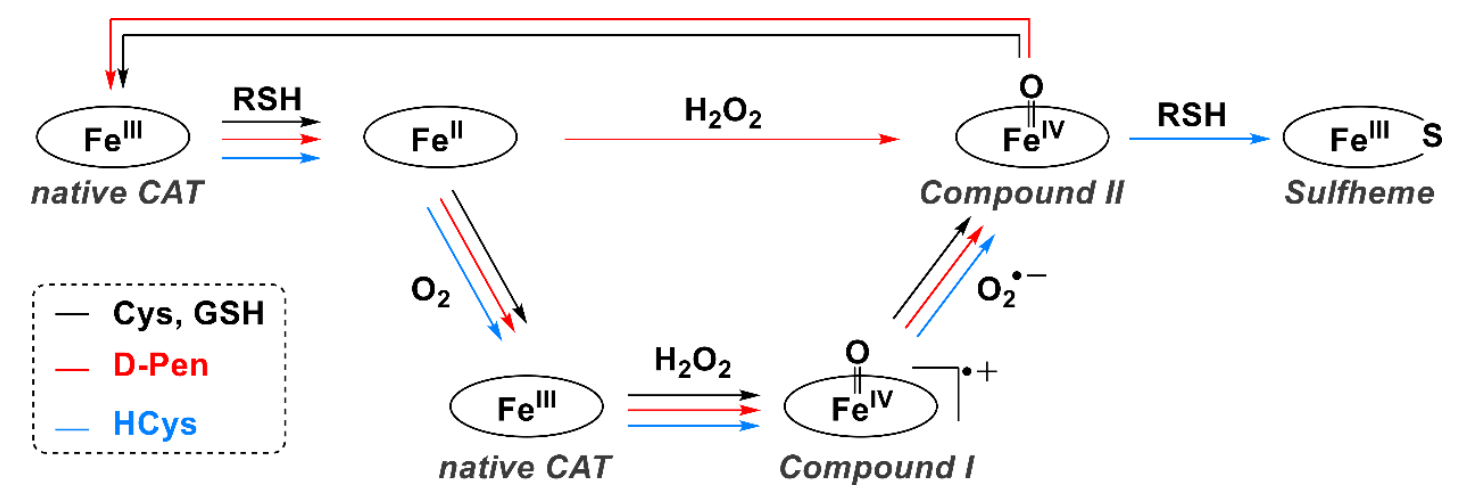

Figure 5 - Reactivity of various biologically relevant thiols toward catalase. 
Due to its chemical properties, D-penicillamine is used as a therapeutic copper chelating agent in the treatment of Wilson's disease (3), as an immunosuppressor in the treatment of rheumatoid arthritis (4), and as a sequestering agent of acetaldehyde in the treatment of alcoholism (9). Unfortunately, adverse effects are fairly frequent with the use of D-penicillamine (10-16), and our study reveals a possible molecular basis for this Janus-faced behavior. Thus, we confirm the pro-oxidant capacity of D-penicillamine that leads to hydrogen peroxide generation by reaction with metal ions, which recently favored its repurposing in anticancer therapy $(\mathbf{2 8 , 2 9 )}$ and may facilitate its action on the acute inflammatory response during treatment of RA (30). In addition, we show that the transient but recurring catalase inactivation initiated by D-Pen could in the long term perturb $\mathrm{H}_{2} \mathrm{O}_{2}$ redox homeostasis and signaling, based on the recent evidence that peroxisomes and catalase are central in the $\mathrm{H}_{2} \mathrm{O}_{2}$ signaling network $(\mathbf{2 3}, \mathbf{4 6})$. Last, a prolonged treatment with D-Pen could induce a chronic inflammatory and oxidative state through chronic disruption of $\mathrm{H}_{2} \mathrm{O}_{2}$ redox homeostasis. This phenomenon could explain the long-term presentations reported in patients treated with D-Pen, that is hepatic iron accumulation and a buildup of iron in the brain, which may be a risk factor for developing neurodegenerative diseases $(\mathbf{1 4 , 4 7 - 5 1 )}$. Thus, iron uptake and cellular accumulation are favored by various $\mathrm{H}_{2} \mathrm{O}_{2}$-dependent mechanisms under pathological conditions, including $\mathrm{H}_{2} \mathrm{O}_{2}$-dependent up-regulation of transferrin receptor 1 (52), stimulation of hepcidin production and ferroportin internalization and degradation $(\mathbf{5 3}, \mathbf{5 4})$, and alteration of ceruloplasmin and its overall ferroxidase activity (55-57).

Funding: This work was in part supported by Idex Université de Paris to D.P.

Acknowledgment: We are indebted to Dr Fabienne Peyrot (Université de Paris, France) for precious assistance with EPR experiments, to Assia Hessani (now at Sorbonne Université, France) for mass-spectrometry analysis, and to Dr Jean-Noël Rebilly (Université Paris-Saclay, France) for useful support with UV-visible stopped-flow experiments. 


\section{Bibliography}

1- Friedman M (1977) Chemical Basis for Pharmacological and Therapeutic Actions of Penicillamine. Proc Roy Soc Med 70: 50-60

2- Joyce DA (1990) D-Penicillamine. Bailliere's Clinical Rheumatology 4: 553-574

3- Brewer GJ, Askari FK (2005) Wilson's disease: clinical management and therapy. J Hepatol 42: S13-S21

4- Suarez-Almazor ME, Spooner C, Belseck E (2000) Penicillamine for treating rheumatoid arthritis. Cochrane Database Syst Rev 4: CD001460

5- Pradhan A, Bagchi A, De S, Mitra S et al (2019) Role of redox imbalance and cytokines in mediating oxidative damage and disease progression of patients with rheumatoid arthritis. Free Radic Res 53:768-779

6- Cuperus RA, Hoogland H, Wever R, Muijsers AO (1987) The effect of D-penicillamine on myeloperoxidase: formation of Compound III and inhibition of the chlorinating activity. Biochim Biophys Acta 912: 124-131

7- Dinis-Oliveira RJ (2016) Oxidative and Non-Oxidative Metabolomics of Ethanol. Curr Drug Metab 17:327-35

8- Nagasawa HT, Goon DJ, DeMaster EG (1978) 2,5,5-Trimethylthiazolidine-4-carboxylic acid, a $\mathrm{D}(-)$-penicillamine-directed pseudometabolite of ethanol. Detoxication mechanism for acetaldehyde. J Med Chem 21: 1274-1279

9- Quertemont E, Didone V (2006) Role of acetaldehyde in mediating the pharmacological and behavioral effects of alcohol. Alcohol Res Health 29: 258-265

10- Deutscher J, Kiess W, Scheerschmidt G, Willgerodt H (1999) Potential hepatotoxicity of penicillamine treatment in three patients with Wilson's disease. J Pediatr Gastroenterol Nutr 29: 628

11- Li J, Uetrecht JP (2009) D-Penicillamine-Induced Autoimmunity: Relationship to Macrophage Activation. Chem Res Toxicol 22: 1526-1533

12- Brewer GJ, Terry CA, Aimsen AM, Hill GM (1987) Worsening of neurologic syndrome in patients with Wilson's disease with initial penicillamine therapy. Arch Neurol 44: 490-493

13- Walshe JM (2011) Penicillamine Neurotoxicity: An Hypothesis. ISRN Neurol 2011: 464572 14- Shiono $\mathrm{Y}$, Wakusawa S, Hayashi $\mathrm{H}$, Takikawa $\mathrm{T}$ et al (2001) Iron accumulation in the liver of male patients with Wilson's Disease. Am J Gastroenterol 96: 3147-3151 
15- Metushi IG, Zhu X, Uetrecht J (2014) D-penicillamine-induced granulomatous hepatitis in brown Norway rats. Mol cell Biochem 393: 229-235

16- Brancaleone V, Esposito I, Gargiulo A, Vellecco V et al (2016) D-Penicillamine modulates hydrogen sulfide $\left(\mathrm{H}_{2} \mathrm{~S}\right)$ pathway through selective inhibition of cystathionine- $\gamma$-lyase. $\mathrm{Br} J$ Pharmacol 173: 1556-1565

17- Stöcker S, Van Laer K, Mijuskovic A, Dick TP (2018) The Conundrum of Hydrogen Peroxide Signaling and the Emerging Role of Peroxiredoxins as Redox Relay Hubs. Antioxid Redox Signal 28: 558-573

18- Sies H (2017) Hydrogen peroxide as a central redox signaling molecule in physiological oxidative stress: Oxidative eustress. Redox Biol 11: 613-619

19- Chen Q, Wang Q, Zhu J, Xiao Q, Zhang L (2018) Reactive oxygen species: key regulators in vascular health and diseases. Br J Pharmacol 175: 1279-1292

20- Dev S, Kumari S, Singh N, Bal SK et al (2015) Role of extracellular Hydrogen peroxide in regulation of iron homeostasis genes in neuronal cells: Implication in iron accumulation. Free Radic Biol Med 86: 78-89

21- Reeder BJ (2010) The Redox Activity of Hemoglobins: From Physiologic Functions to Pathologic Mechanisms. Antioxid Redox Signal 13: 1087-1123

22- Padovani D, Hessani A, Castillo FT, Liot G et al (2016) Sulfheme formation during homocysteine S-oxygenation by catalase in cancers and neurodegenerative diseases. Nat Commun 7: 13386

23- Lismont C, Nordgren M, Brees C, Knoops B, Van Veldhoven PP, Fransen M (2019) Peroxisomes as Modulators of Cellular Protein Thiol Oxidation: A New Model System. Antioxid Redox Signal 30:22-39

24- Nicholls P (2012) Classical catalase: ancient and modern. Arch Biochem Biophys 525: 95101

25- Lardinois OM (1995) Reactions of bovine liver catalase with superoxide radicals and hydrogen peroxide. Free Rad Res 22: 251-274.

26- Bandara DM, Sono M, Bruce GS, Brash AR, Dawson JH (2011) Coordination modes of tyrosinate-ligated catalase-type heme enzymes: magnetic circular dichroism studies of Plexaura homomalla allene oxide synthase, Mycobacterium avium ssp. paratuberculosis protein-2744c, and bovine liver catalase in their ferric and ferrous states. J Inorg Biochem 105: 1786-1794 
27- Hoogenboom R (2010) Thiol-yne chemistry: a powerful tool for creating highly functional materials. Angew Chem Int Ed Engl 49: 3415-3417

28- Gupte A, Mumper RJ (2007) An investigation into copper catalyzed D-penicillamine oxidation and subsequent hydrogen peroxide generation. $J$ Inorg Biochem 101:594-602

29- Gupte A, Mumper RJ (2007) Copper chelation by D-penicillamine generates reactive oxygen species that are cytotoxic to human leukemia and breast cancer cells. Free Radic Biol Med 43:1271-1278

30- Starkebaum G, Root RK (1985) D-Penicillamine: analysis of the mechanism of coppercatalyzed hydrogen peroxide generation. J Immunol 134:3371-3378

31- Nagababu E, Rifkind JM (2004) Heme degradation by reactive oxygen species. Antioxid Redox Signal 6: 967-978

32- Jantschko W, Furtmüller PG, Zederbauer M, Lanz M, Jakopitsch C, Obinger C (2003) Direct conversion of ferrous myeloperoxidase to compound II by hydrogen peroxide: an anaerobic stopped-flow study. Biochem Biophys Res Commun 312:292-298

33- Jantschko W, Furtmüller PG, Zederbauer M, Neugschwandtner K, Jakopitsch C, Obinger C (2005) Reaction of ferrous lactoperoxidase with hydrogen peroxide and dioxygen: an anaerobic stopped-flow study. Arch Biochem Biophys 434:51-59

34- Takeda A, Miyahara T, Hachimori A, Samejima T (1980) The interactions of thiol compounds with porcine erythrocyte catalase. J Biochem 87: 429-439

35- Millar F, Wrigglesworth JM and Nicholls, P (1981) Ligand binding to catalase and metmyoglobin. Direct measurements of proton involvement. Eur J Biochem 117: 13-17

36- Nicholls P, Fita I, Loewen PC (2000) Enzymology and structure of catalases. Adv Inorg Chem 51: 51-106

37- Nicholls P (2012) Classical catalase: ancient and modern. Arch Biochem Biophys 525: 95101

38- Winterbourn CC, Metodiewa D (1999) Reactivity of biologically important thiol compounds with superoxide and hydrogen peroxide. Free Radic Biol Med 27: 322-328

39- Bauer G (2015) Increasing the endogenous NO level causes catalase inactivation and reactivation of intercellular apoptosis signaling specifically in tumor cells. Redox Biol 6: 353371

40- Nicholls $P$ (1964) The reaction of azide with catalase and their significance. Biochem $J$ 90:331-343 
41- Kalyanaraman B, Janzen EG, Mason RP (1985) Spin trapping of the azidyl radical in azide/catalase/H2O2 and various azide/peroxidase/H2O2 peroxidizing systems. J Biol Chem 260:4003-4006

42- Wiesner K (1962) The reaction mechanism of catalase. Experientia 18: 115-116 43- Nicholls $P$ (1963) Ferrous complexes in the catalase reaction. Experientia 19: 80-82 44- Hioe J, Zipse H (2010) Radical stability and its role in synthesis and catalysis. Org Biomol Chem 8: 3609-3617

45- Degirmenci I, Coote ML (2016) Effect of Substituents on the Stability of Sulfur-Centered Radicals. J Phys Chem A 120: 7398-403

46- Lismont C, Revenco J, Fransen M (2019) Peroxisomal Hydrogen Peroxide Metabolism and Signaling in Health and Disease. Int J Mol Sci 20: 3673

47- Rodriguez-Castro KI, Hevia-Urrutia FJ, Sturniolo GC (2015) Wilson's disease: A review of what we have learned. World J Hepatol 7: 2859-2870

48- Pfeiffenberger J, Gotthardt DN, Herrmann T et al (2012) Iron metabolism and the role of HFE gene polymorphisms in Wilson's disease. Liver Int 32: 165-170

49- Litwin T, Gromadzka G, Czlonkowska A (2012) Gender differences in Wilson's Disease. J Neurol Sci 312: 31-35

50- Pak K, Ordway S, Sadowski B, Canevari M, Torres D (2021) Wilson's Disease and Iron Overload: Pathophysiology and Therapeutic Implications. Clin Liver Dis 17:61-66

51- Medici V, Di Leo V, Lamboglia F, Bowlus CL et al (2007) Effect of penicillamine and zinc on iron metabolism in Wilson's disease. Scand J Gastroenterol 42:1495-500

52- Andriopoulos B, Hegedüsch S, Mangin J, Riedel HD, Hebling U, Wang J, Pantopoulos K, Mueller S (2007) Sustained hydrogen peroxide induces iron uptake by transferrin receptor-1 independent of the iron regulatory protein/iron-responsive element network. $J$ Biol Chem 282:20301-20308

53- Millonig G, Ganzleben I, Peccerella T, Casanovas G, Brodziak-Jarosz L, Breitkopf-Heinlein K, Dick TP, Seitz HK, Muckenthaler MU, Mueller S (2012) Sustained submicromolar H2O2 levels induce hepcidin via signal transducer and activator of transcription 3 (STAT3). J Biol Chem 287:37472-37482

54- Schmidt PJ (2015) Regulation of Iron Metabolism by Hepcidin under Conditions of Inflammation. J Biol Chem 290:18975-83 
55- Vasilyev VB (2019) Looking for a partner: ceruloplasmin in protein-protein interactions. Biometals 32:195-210

56- Choi SY, Kwon HY, Kwon OB, Eum WS, Kang JH (2000) Fragmentation of human ceruloplasmin induced by hydrogen peroxide. Biochimie 82(2):175-80

57- Aouffen M, Paquin J, Furtos A, Waldron KC, Mateescu MA (2004) Oxidative aggregation of ceruloplasmin induced by hydrogen peroxide is prevented by pyruvate. Free Radic Res 38:19-26 